\title{
PRONÓSTICO DE VIDA A TRES MESES Y FACTORES ASOCIADOS DE PACIENTES CON ALTERACIÓN DEL ESTADO MENTAL EN EMERGENCIA DE MEDICINA DE UN HOSPITAL NACIONAL EN PERÚ
}

\author{
Delia Alva-Rodriguez,a, María de los Ángeles Lazo²,a, Javier D. Loza-Herrera²,a, Germán Málaga1,2,b
}

\begin{abstract}
RESUMEN
Objetivos. Determinar el pronóstico de vida a tres meses y factores asociados en pacientes con alteración del estado mental (AEM). Materiales y métodos. Estudio transversal analítico que incluyó pacientes mayores de 18 años admitidos por emergencia de medicina de un hospital nacional, con AEM, dentro de las veinticuatro horas de iniciado el cuadro y que requirieron observación. La información demográfica, clínica y valoración funcional fueron tomadas de historias clínicas. El seguimiento a los tres meses se hizo por vía telefónica. Resultados. Se incluyó a 290 pacientes. Esta entidad representa el $4,1 \%$ del total de atenciones de emergencia. La mortalidad global fue $24,2 \%$, mientras que en el subgrupo de adultos mayores fue de $28,1 \% ; 19,3 \%$ en adultos y $15,2 \%$ en adultos jóvenes. La edad promedio fue 63,5 años y el grupo de adultos mayores constituyó casi $2 / 3$ de la población. El $51 \%$ estuvo compuesto por varones. El promedio de estancia hospitalaria fue de 7 días. Las causas asociadas, más frecuentes, fueron: enfermedades infecciosas $(44,1 \%)$, neurológicas $(40,3 \%)$, respiratorias $(28,3 \%)$, metabólicos $(18,6 \%)$ y cardiovasculares $(17,2 \%)$. La asociación de mortalidad con nivel $<7$ en la ECG tuvo una RP=2,9 $p<0,001 y$ el de funcionalidad con puntaje de 5 en la escala modificada de Rankin una $\mathrm{RP}=8,2 p=0,03$. Conclusión. La AEM, presente en el $4 \%$ de las admisiones por emergencia, se asocia a mortalidad en una de cada cuatro personas. La causa predominante lo constituyen las enfermedades infecciosas. El compromiso de la funcionalidad, fue el principal predictor de mortalidad.
\end{abstract}

\section{THREE-MONTH LIFE PROGNOSIS AND ASSOCIATED FACTORS IN PATIENTS WITH ALTERED MENTAL STATUS ADMIMTTED TO THE EMERGENCY ROOM OF A NATIONAL HOSPITAL IN PERU}

\begin{abstract}
Objectives. Determine the prognosis at three months and associated factors of patients with altered mental status (AMS). Materials and methods. Cross-sectional study that included patients over 18 years of age admitted to the emergency room in a national hospital, with AMS, within twenty-four hours of onset and that required observation. The demographic, clinical and functional assessment information were taken from the medical record. A follow-up was made at three months by telephone. Results. The study included 290 patients, representing $4.1 \%$ of the total patients in the emergency room. Overall mortality was $24.2 \%$, whereas in the subgroup of older adults was $28.1 \% ; 19.3 \%$ in adults and $15.2 \%$ in young adults. The average age was 63.5 years and the elderly group constituted nearly two thirds of the population. $51 \%$ were males. The average hospital stay was 7 days. The most frequently associated causes were infectious diseases (44.1\%), neurological $(40.3 \%)$, respiratory $(28.3 \%)$, metabolic $(18.6 \%)$, and cardiovascular $(17.2 \%)$ disorders. Mortality was associated with a) GCS levels $<7$ (OR 2.9, p<0.001) and b) functionality score of 5 on the modified Rankin scale (OR 8.2, $p=0.03)$. Conclusions. AMS, present in $4 \%$ of emergency admissions, is associated with mortality in one in four people. The predominant cause infectious diseases. The commitment of the functionality was the main predictor of mortality.
\end{abstract}

Key words: Mental health assistance; Etiology; Causality; Mortality (source: MeSH/NLM).

\footnotetext{
Hospital Nacional Cayetano Heredia. Lima, Perú.

Unidad de Conocimiento y Evidencia, Universidad Peruana Cayetano Heredia. Lima, Perú.

Médico cirujano; ${ }^{\mathrm{b}}$ médico internista, magíster en Medicina

Recibido: 24-11-13 Aprobado: 06-08-14
}

Citar como: Alva-Rodriguez D, Lazo MA, Loza-Herrera JD, Málaga G. Pronóstico de vida a tres meses y factores asociados de pacientes con alteración del estado mental en emergencia de medicina de un hospital nacional en Perú. Rev Peru Med Exp Salud Publica. 2014;31(3):480-86. 


\section{INTRODUCCIÓN}

La alteración del estado mental (AEM) es una condición clínica frecuente que representa entre 4 a $10 \%$ de las atenciones en los servicios de emergencia ${ }^{(1,2)}$ y esta constituida por una serie de etiologías, por lo que la estrategia diagnóstica y la terapia de emergencia son variadas y deben ser decididas en el mismo momento $(2,3)$. Esta condición comprende condiciones clínicas y estados patológicos como delirio, estado confusional agudo y alteración del nivel y contenido de la conciencia (3-5), que se asocian a causas diversas, genera elevada estancia hospitalaria, morbilidad importante, discapacidad de grado diverso y mortalidad ${ }^{(6-8)}$. Si a esto le agregamos que los pacientes con discapacidad adquirida, tendrían pobre adherencia en el seguimiento, según lo hallado en un estudio, aún en revisión, realizado en pacientes seguidos después de su "primer accidente cerebrovascular", donde más del $50 \%$ se perdió en el seguimiento; son indicativos de que la AEM, representa un severo impacto para el sistema de salud ${ }^{(9)}$.

Estudios realizados en los departamentos de emergencia en países de altos ingresos, describen como causas más frecuentes de AEM: hipoglucemia, accidente cerebrovascular, intoxicaciones, infecciones y menos frecuentemente entidades como trastornos metabólicos, encefalopatía hepática y psicosis ${ }^{(2-8,10)}$. No se halló información con relación a la prevalencia de entidades causantes de AEM en países de medianos o bajos ingresos.

En general, no se dispone de información relacionada con el seguimiento al alta, en términos de adherencia a tratamiento, secuelas en la funcionalidad, rehabilitación, morbilidad, soporte a cuidadores, rehospitalizaciones y mortalidad, en pacientes con AEM, pues los trabajos encontrados en la literatura son heterogéneos en cuanto a clasificación de etiologías, limitados a plazos cortos de seguimiento clínico, incompletos en cuanto a desenlaces registrados y realizados en áreas geográficas completamente diferente a la del Perú e incluso la región, por lo que no se tiene información que permita de manera confiable, elaborar protocolos, guías de manejo, asignación de recursos ni evaluación de lo que representa como impacto económico para el sistema de salud y la sociedad ${ }^{(5-6)}$.

Por lo expuesto, se planteó hacer un estudio que permitiera determinar las causas más frecuentes de alteración del estado mental, en pacientes que ingresaron a la emergencia de medicina de un hospital nacional, conocer la mortalidad hospitalaria y a tres meses de seguimiento, sus factores asociados, los que además permita hacer las correspondientes intervenciones para mejorar la situación, estableciendo a partir de este estudio, una línea de investigación.

\section{MATERIALES Y MÉTODOS}

\section{DISEÑO DE ESTUDIO}

Se realizó un estudio descriptivo transversal analítico, en pacientes adultos que ingresaron con AEM a la emergencia de medicina de un hospital nacional entre diciembre 2011 y febrero del 2012 por muestreo consecutivo con cobertura al $100 \%$.

\section{TAMAÑO MUESTRAL}

Fue calculado usando una proporción estimada de mortalidad temprana en pacientes con AEM de 12,5\% y un límite de confianza de $95 \%$, se obtuvo un mínimo tamaño muestral de 263 individuos ${ }^{(2,5,6)}$.

\section{CRITERIOS DE SELECCIÓN}

Se seleccionó un período al azar que se estimó en el que se pudieran reclutar dicha cantidad de pacientes (3 meses). Se incluyó a pacientes que presentaron (AEM) de menos de $24 \mathrm{~h}$ de evolución y que por su condición requirieran permanecer en observación. Se excluyó a pacientes cuya causa de la AEM fuera de origen traumático, pues en ese caso, la causa de la AEM, era obvia y, por lo tanto, fuera del alcance del objetivo del estudio, pacientes con alteración crónica del estado mental que no hubieran presentado cambios desde su estado basal en las últimas veinticuatro horas y pacientes derivados de otra institución (hospital, centro de salud o equivalentes) que hubieran recibido manejo médico previo, incluso si esto ocurría antes de $24 \mathrm{~h}$.

\section{PROCEDIMIENTOS PARA LA OBTENCIÓN DE INFORMACIÓN}

Los pacientes fueron seleccionados del reporte diario de emergencias, en el que se identificó a pacientes que ingresaron con alteración del estado mental, además, se revisó cada una de las historias de la emergencia para identificar casos omitidos en el reporte diario, en donde se verificó que en los diagnósticos de ingreso figurara el diagnóstico de alteración del estado mental o alguno de sus componentes (alteración del nivel de conciencia, alteración del contenido de la conciencia, delirio) o términos que pudieran expresar sinonimia como deterioro del estado mental, alteración de nivel y contenido de estado mental, entre otros. Se obtuvo información sociodemográfica, de comorbilidades relevantes para el estudio como hipertensión arterial, diabetes mellitus, accidente cerebrovascular, enfermedad cardiaca, cáncer, trastornos psiquiátricos, enfermedad renal crónica, uso de medicamentos o sustancias ilícitas; diagnósticos de ingreso, y situación del estado funcional al ingreso. Se hizo seguimiento al alta, de donde 
se obtuvo información de la condición de egreso y funcionalidad. Posteriormente, a los tres meses, se realizó el seguimiento telefónico en una oportunidad, de donde que se recolectó información del paciente, y si este no podía comunicarse del cuidador; en este contacto se indagó por el estado vital del paciente y su estado funcional.

Para medir la funcionalidad se aplicó la escala de coma de Glasgow ${ }^{(11)}$ y la escala modificada de Rankin (EmR), herramienta utilizada para medir discapacidad y proporciona un índice global de salud (12,13). Esta herramienta ha demostrado utilidad en estudios multicéntricos, permitiendo evaluar el pronóstico de los pacientes neurológicos críticos, principalmente con compromiso cerebrovascular; la puntuación se realiza de 0 a 6 , correspondiendo 0 : asintomático; 1 : no discapacidad significativa; 2: discapacidad leve; 3: discapacidad moderada; 4: moderada a severa discapacidad; 5: incapacidad grave, y 6: muerte. La evaluación de seguimiento fue hecha por vía telefónica y se comparó con los valores del ingreso y alta hospitalaria, la información relacionada con la valoración fue tomada de la nota de evaluación del neurólogo y, si no hubiera sido consignada, se procedía a solicitarla explícitamente ${ }^{(14-17)}$.

\section{ANÁLISIS ESTADÍSTICO}

Fue realizado en el programa estadístico STATA 12 (StataCorp, Texas). Se describió la proporción de pacientes con los antecedentes explorados y grupos de diagnóstico mediante proporciones e intervalos de confianza, esta descripción se agrupó de acuerdo con la edad en tres grupos poblaciones que incluían a jóvenes (18-39 años), adultos (40-59 años) y adultos mayores (>60 años).

Se estimó la variable principal de estudio "mortalidad al tercer mes de la hospitalización," mediante una proporción e intervalo de confianza, además, se realizó un estudio exploratorio de posibles asociaciones de la mortalidad con: antecedentes, funcionalidad al ingreso usando la escala modificada de Rankin (asociado a un puntaje mayor o igual a 5) y el puntaje en la escala de Glasgow al ingreso (expresado como la proporción de pacientes con Glasgow $<7$ de la ECG), usando razones de prevalencias (RP) e intervalos de confianza, ajustadas para edad y sexo, además, se realizó una valoración de la funcionalidad al ingreso y a los tres meses de seguimiento. La calificación se estratificó en: igual, mejor o peor, considerando un cambio de 1 punto en la escala de Rankin.

\section{CONSIDERACIONES ÉTICAS}

El presente trabajo contó con la aprobación de los comités de ética del Hospital Nacional Cayetano Heredia y el de la Universidad Peruana Cayetano Heredia.

\section{RESULTADOS}

Se evaluó a 300 pacientes, de los cuales 290 fueron incluidos con alteración del nivel del estado mental. Fueron excluidos diez pacientes (uno por ser la AEM de etiología neuroquirúrgica y nueve por haber recibido tratamiento previo en otra institución y haber arribado

Tabla 1. Características generales de los pacientes que ingresaron con alteración del nivel de conciencia

\begin{tabular}{|c|c|c|c|c|}
\hline \multirow{2}{*}{ Características } & Adulto joven & Adulto & Adulto mayor & Todos \\
\hline & $\mathrm{N}=50(\%)$ & $\mathrm{N}=58(\%)$ & $\mathrm{N}=182(\%)$ & $\mathrm{N}=290(\%)$ \\
\hline Sexo femenino (\%) & $12(24,0)$ & $27(46,6)$ & $103(56,6)$ & $142(49,0)$ \\
\hline \multicolumn{5}{|l|}{ Instrucción } \\
\hline Analfabetos & $1(2,0)$ & $4(6,90)$ & $39(21,4)$ & $44(15,2)$ \\
\hline Primaria & $10(20,0)$ & $17(29,3)$ & $96(52,8)$ & $123(42,4)$ \\
\hline Secundaria & $23(46,0)$ & $22(37,9)$ & $35(19,2)$ & $80(27,6)$ \\
\hline Superior & $14(28,0)$ & $13(22,4)$ & $11(6,0)$ & $38(13,1)$ \\
\hline Sin datos & $2(4,0)$ & $2(3,5)$ & $1(0,6)$ & $5(1,72)$ \\
\hline \multicolumn{5}{|l|}{ Ocupación } \\
\hline Estudiante & $8(16,0)$ & $0(0,0)$ & $1(0,55)$ & $9(3,10)$ \\
\hline Empleado & $23(46,0)$ & $28(48,3)$ & $18(9,9)$ & $69(23,8)$ \\
\hline Desocupado temporal & $16(32,0)$ & $27(46,6)$ & $16(8,8)$ & $59(20,3)$ \\
\hline Jubilado/Pensionado & $0(0,0)$ & $0(0,0)$ & $11(3,8)$ & $11(3,8)$ \\
\hline Otros inactivos & $3(6,0)$ & $3(5,2)$ & $136(74,7)$ & $142(49,0)$ \\
\hline \multicolumn{5}{|l|}{ Funcionalidad basal (Rankin) } \\
\hline Puntaje 0-2 & $34(68,0)$ & $26(44,8)$ & $43(23,6)$ & $103(35,5)$ \\
\hline Puntaje 3-4 & $16(32,0)$ & $30(51,7)$ & $120(65,9)$ & $166(57,2)$ \\
\hline Puntaje 5 & $0(0,0)$ & $2(3,5)$ & $19(10,4)$ & $21(7,2)$ \\
\hline Puntaje $<8$ & $8(16,0)$ & $3(5,2)$ & $17(9,5)$ & $28(9,8)$ \\
\hline Puntaje 8-13 & $20(40)$ & $21(36,1)$ & $95(53,1)$ & $136(47,4)$ \\
\hline Puntaje > 13 & $22(44)$ & $34(58,6)$ & $67(37,4)$ & $123(42,9)$ \\
\hline \multicolumn{5}{|l|}{ Antecedentes } \\
\hline Hipertensión arterial & $1(2,0)$ & $10(17,2)$ & $80(44,0)$ & $91(31,4)$ \\
\hline Diabetes mellitus & $4(8,0)$ & $6(10,3)$ & $44(24,6)$ & $54(18,6)$ \\
\hline Infección por VIH & $13(26,0)$ & $2(3,4)$ & $1(0,6)$ & $16(5,5)$ \\
\hline ACV previo & $0(0,0)$ & $4(6,9)$ & $15(8,2)$ & $19(6,6)$ \\
\hline Enfermedad cardiaca & $0(0,0)$ & $3(5,2)$ & $5(2,8)$ & $8(2,8)$ \\
\hline Enfermedad renal crónica & $0(0,0)$ & $4(6,9)$ & $9(5,0)$ & $13(4,5)$ \\
\hline
\end{tabular}


Tabla 2. Diagnósticos generales de la muestra*

\begin{tabular}{|c|c|c|c|c|}
\hline \multirow{2}{*}{ Diagnósticos } & Adulto joven & Adulto & Adulto mayor & Total \\
\hline & $\mathrm{N}=50$ & $\mathrm{~N}=58$ & $\mathrm{~N}=182$ & $\mathrm{~N}=\mathbf{2 9 0}$ \\
\hline Infecciones de vías respiratorias & $22,0 \%$ & $17,2 \%$ & $29,7 \%$ & $25,9 \%$ \\
\hline Sepsis y choque séptico & $6,0 \%$ & $10,3 \%$ & $21,9 \%$ & $16,9 \%$ \\
\hline Accidente vascular isquémico & $2,0 \%$ & $17,2 \%$ & $15,9 \%$ & $13,8 \%$ \\
\hline Accidente vascular hemorrágico & $2,0 \%$ & $8,6 \%$ & $9,9 \%$ & $8,3 \%$ \\
\hline Insuficiencia cardiaca descompensada & $0,0 \%$ & $13,8 \%$ & $16,5 \%$ & $13,1 \%$ \\
\hline Infección de vías urinarias & $6,0 \%$ & $8,6 \%$ & $12,1 \%$ & $10,3 \%$ \\
\hline Encefalopatías metabólicas & $4,0 \%$ & $20,7 \%$ & $5,5 \%$ & $8,3 \%$ \\
\hline Insuficiencia respiratoria & $2,0 \%$ & $5,2 \%$ & $8,8 \%$ & $6,9 \%$ \\
\hline Trastorno hidroelectrolítico & $2,0 \%$ & $1,7 \%$ & $9,3 \%$ & $6,6 \%$ \\
\hline Infecciones del sistema nervioso central & $12,0 \%$ & $5,2 \%$ & $2,7 \%$ & $4,8 \%$ \\
\hline Convulsiones secundarias & $10,0 \%$ & $8,6 \%$ & $2,2 \%$ & $4,8 \%$ \\
\hline Neoplasias & $8,0 \%$ & $0,0 \%$ & $3,9 \%$ & $3,8 \%$ \\
\hline Convulsiones por epilepsia & $2,0 \%$ & $1,7 \%$ & $1,6 \%$ & $3,5 \%$ \\
\hline Hiperglicemias & $6,0 \%$ & $1,7 \%$ & $2,7 \%$ & $3,1 \%$ \\
\hline Hemorragia Digestiva & $2,0 \%$ & $6,9 \%$ & $2,2 \%$ & $3,1 \%$ \\
\hline Confusión aguda & $0,0 \%$ & $1,7 \%$ & $3,8 \%$ & $2,7 \%$ \\
\hline Tumor cerebral & $12,0 \%$ & $0,0 \%$ & $2,2 \%$ & $2,7 \%$ \\
\hline Enfermedad psiquiátrica & $10,0 \%$ & $1,7 \%$ & $1,1 \%$ & $2,7 \%$ \\
\hline Encefalopatía hipertensiva & $0,0 \%$ & $1,7 \%$ & $3,3 \%$ & $2,4 \%$ \\
\hline Hipoglicemias & $0,0 \%$ & $3,5 \%$ & $2,2 \%$ & $2,1 \%$ \\
\hline Síndrome coronario agudo & $0,0 \%$ & $1,7 \%$ & $1,7 \%$ & $1,4 \%$ \\
\hline Infecciones gastrointestinales & $0,0 \%$ & $1,7 \%$ & $1,7 \%$ & $1,4 \%$ \\
\hline Intoxicaciones por gesto suicida & $4,0 \%$ & $0,0 \%$ & $0,0 \%$ & $0,7 \%$ \\
\hline Intoxicación alcohol & $0,0 \%$ & $0,0 \%$ & $1,1 \%$ & $0,7 \%$ \\
\hline Intoxicación por fármacos & $2,0 \%$ & $0,0 \%$ & $0,0 \%$ & $0,3 \%$ \\
\hline
\end{tabular}

* Hubo pacientes que estuvieron clasificados en más de una categoría, por lo que la sumatoria es mayor al $100 \%$

después de $24 \mathrm{~h}$ ). Esta entidad representó el 4,1\% de la totalidad de admisiones de emergencia al servicio de medicina. El promedio de edad de la población fue de 63,5 años $\pm 20,7$ (62,8\% fueron adultos mayores). La proporción de varones constituyó el 51\% (Tabla 1).

En cuanto a los antecedentes, las enfermedades crónicas no transmisibles predominaron en los adultos mayores, mientras que las enfermedades crónicas infecciosas, en particular infección por VIH (26,6\%) en adultos jóvenes, se halló, además, una asociación a mortalidad en este grupo población ajustado por edad y sexo (RP 3,7; IC 95\%: 0,98-14,49; p<0,053) (Tabla 1).

La estancia hospitalaria promedio, fue de 7 días (rango intercuartíl 3-10 días), sin diferencias entre ambos sexos.

Las causas identificadas de AEM determinadas en la evaluación al alta, fueron agrupadas en grupos de enfermedades, por lo que un mismo paciente pudiera haber sido catalogado en más un grupo. Las infecciones constituyeron el 44,1\% (4,8\% meningoencefalitis), causas neurológicas en $40,3 \%$ y enfermedades respiratorias en $28,3 \%$. En menor proporción se encontró trastornos metabólicos (18,6\%), enfermedades cardiovasculares $(17,2 \%)$, enfermedades digestivas $(10,3 \%)$, intoxicaciones $(3,8 \%)$ y otras como tumores y enfermedades psiquiátricas (depresión, cuadros psicóticos e intoxicación por fármacos) $(6,6 \%)$. Los detalles se observan en la Tabla 2 y 3.

En el subgrupo de adultos mayores, las causas más frecuentes fueron las infecciones $(49,5 \%)$ dentro de estos principalmente vías respiratorias $(29,6 \%)$, urinarias $(12,1 \%)$ y meningoencefalitis $(2,7 \%)$; seguido de las causas neurológicas de etiología vascular (38,5\%), enfermedades respiratorias $(38,5 \%)$ y enfermedades cardiovasculares $(22,5 \%)$.

En el subgrupo de adultos se halló que las causas más frecuentes son: neurológicas $41,4 \%$, infecciosas $32,8 \%$, (5,2\% fueron meningoencefalitis), y trastornos metabólicos en $25,9 \%$, mientras que en el subgrupo de adulto joven el principal motivo de ingreso fueron los cuadros neurológicos 46,0\% (convulsiones $(22,0 \%)$, tumores del SNC $(12,0 \%)$, y meningoencefalitis $(12,0 \%)$ Tabla 3.

La mortalidad hospitalaria fue $22,7 \%$ (68 pacientes) Tabla 4. A los tres meses de seguimiento, se constató el fallecimiento de cuatro pacientes, con lo que la 
Tabla 3. Diagnósticos de egreso de los pacientes con alteración del estado nivel de conciencia

\begin{tabular}{|c|c|c|c|c|}
\hline \multirow{2}{*}{ Características } & Adulto joven & Adulto & Adulto mayor & Todos \\
\hline & $\mathrm{N}=50(\%)$ & $\mathrm{N}=58(\%)$ & $\mathrm{N}=182(\%)$ & $\mathrm{N}=290(\%)$ \\
\hline Infecciones & $19(38,0)$ & $19(32,8)$ & $90(49,5)$ & $128(44,1)$ \\
\hline Neurológicas & $23(46,0)$ & $24(41,4)$ & $70(38,5)$ & $117(40,3)$ \\
\hline Respiratorias & $11(22,0)$ & $12(20,7)$ & $59(32,4)$ & $82(28,4)$ \\
\hline Trastornos metabólicos & $6(12,0)$ & $15(25,9)$ & $33(18,1)$ & $54(18,6)$ \\
\hline Cardiovasculares & $0(0,0)$ & $9(15,5)$ & $41(22,5)$ & $50(17,2)$ \\
\hline Renales & $4(8,0)$ & $6(10,3)$ & $26(14,3)$ & $36(12,4)$ \\
\hline Abdominales & $2(4,0)$ & $15(25,9)$ & $13(7,1)$ & $30(10,3)$ \\
\hline Otros (tumorales y psiquiátricas) & $9(18,0)$ & $1(1,7)$ & $9(5,0)$ & $19(6,6)$ \\
\hline Intoxicaciones & $6(12,0)$ & $2(3,5)$ & $3(17,7)$ & $11(3,8)$ \\
\hline
\end{tabular}

* Hubo pacientes que estuvieron clasificados en más de una categoría, por lo que la sumatoria es mayor al $100 \%$

mortalidad acumulada fue de $24,2 \%$. La mortalidad en el subgrupo de adulto mayor fue $28,1 \%$.

Adicionalmente, se halló que $8 \%$ (18/222) de pacientes fueron rehospitalizados en los tres meses de seguimiento. Se constató el estado vital en todos los pacientes, pero en diez de ellos no se pudo hacer la valoración de funcionalidad.

Hubo relación entre afección severa de la funcionalidad y mortalidad: los pacientes con EmR compromiso grave (puntaje 5) tuvieron una RP 8,2 (IC $95 \%$ : $1,28-57,87 ; p=0,03)$ ajustado por edad y sexo y los pacientes con ECG $<7$ al ingreso tuvieron un RP 2,9 (IC 95\%: 1,70-5,18; $p<0,001$ ) ajustado por edad y sexo. No se halló correlación con las demás variables analizadas.

Las enfermedades que se encontraron más frecuentemente en los pacientes fallecidos fueron las infecciones (principalmente de las vías respiratorias) con $35 \%$; los accidentes cerebrovasculares con $27 \%$ (correspondiendo $13,7 \%$ a $\mathrm{ACV}$ isquémico, 6,5\% hemorrágico y $3,7 \%$ hemorragia subaracnoidea y $3,1 \%$ compromiso mixto, es decir, isquémico hemorrágico), y compromiso pulmonar complicado con insuficiencia respiratoria con $25 \%$. De las 50 muertes ocurridas entre los adultos mayores, el
$28,1 \%$ (14) se debieron a compromiso pulmonar con insuficiencia respiratoria.

\section{DISCUSIÓN}

La alteración del estado mental es una condición frecuente en emergencia que en nuestro estudio se asoció a una mortalidad de $24,2 \%$, es decir, uno de cada cuatro pacientes que ingresan en esta condición fallecen antes de los tres meses. La mortalidad por esta entidad, duplica la mortalidad global del mismo hospital, reportada en $12 \%{ }^{(18)}$. No se halló series locales o latinoamericanas que hubieran hecho esta evaluación, así que solo se puede comparar estos hallazgos con series realizadas de países desarrollados donde la mortalidad reportada fue alrededor del $10 \%{ }^{(2,5,19,20)}$. Estos resultados permiten inferir que las personas que se presentan con AEM en emergencia tienen un pronóstico de mortalidad inusitadamente alto, mucho más del correspondiente a su respectivo grupo etario, haciendo impostergable dirigir esfuerzos y priorizar su atención, así como en las causas potencialmente tratables para disminuir esta complicación de alta letalidad.

En cuanto a los predictores de mortalidad en estos pacientes, estuvo especialmente relacionada con el

Tabla 4. Valoración de la funcionalidad al alta de los pacientes con alteración del nivel de conciencia a los tres meses del alta (escala de Rankin modificada)

\begin{tabular}{|c|c|c|c|c|}
\hline \multirow{2}{*}{ Características } & Adulto joven & Adulto & Adulto mayor & Todos \\
\hline & $\mathrm{N}=46(\%)$ & $\mathrm{N}=57(\%)$ & $\mathrm{N}=178(\%)$ & $\mathrm{N}=281(\%)$ \\
\hline Igual & $11(24,0)$ & $21(36,8)$ & $73(41,0)$ & $105(37,4)$ \\
\hline Mejoró & $2(4,4)$ & $1(1,8)$ & $0(0,0)$ & $3(1,1)$ \\
\hline Empeoró & $26(56,5)$ & $24(42,1)$ & $55(30,9)$ & $105(37,4)$ \\
\hline Falleció & $7(15,2)$ & $11(19,3)$ & $50(28,1)$ & $68(24,2)$ \\
\hline
\end{tabular}


grado de compromiso mostrado en la ECG y la EmR (correspondiendo el compromiso funcional moderado y severo el $60 \%$ de mortalidad). Esta es información no disponible en otros estudios, pues no se halló quienes hayan hecho previamente esta evaluación. Con relación a este punto se debe considerar que no se encontró correlación lineal al comparar ambas variables en el incremento de la mortalidad, pudiéndose inferir que ambas variables, de por sí, son determinantes independientes de mortalidad y mal pronóstico.

La $A E M$, es una entidad frecuente, que representó el $4,1 \%$ de todos las admisiones de pacientes por emergencia, promedio similar al reportado en la literatura internacional, entre 1,3 y $5 \%{ }^{(1-5,14)}$, esto, a pesar de las objeciones realizadas en torno la diferente procedencia de la literatura hallada.

El subgrupo de pacientes más afectado correspondió al grupo adulto mayor $(62,8 \%)$, hallazgo similar a lo reportado por trabajos realizados en Singapur y Taiwán (5-6) donde los adultos mayores constituyeron más del $60 \%$ de la población que ingreso por AEM, mientras que en otro trabajo realizado por William Kanich et al., la edad promedio fue de 49 años ${ }^{(2)}$.

En este estudio se encontró que las causas más frecuentes deAEM las constituyeron las enfermedades infecciosas, que predominaron en el grupo adulto mayor $(44,1 \%)$, resultado que difiere con lo reportado en la literatura internacional, en la que predominan etiologías vasculares primarias del SNC y las causas metabólicas; sin embargo, como se comentó, la literatura referida proviene de países de altos ingresos, en este punto se debe reafirmar la naturaleza pionera de este estudio, pues no se halló información nacional, o regional, contra la que podamos contrastar estos hallazgos ${ }^{(2,5-7)}$. Esto podría sugerir que, si bien está ocurriendo lo que se llama un periodo de transición epidemiológica, en que las enfermedades crónicas no comunicables son cada vez más importantes en el Perú, las enfermedades infecciosas tienen aún un papel no desdeñable y, de otro lado, la correlación existente y descrita entre obesidad y diabetes y mayor severidad de infecciones, y mayor mortalidad de enfermedades infecciosas como neumonía, es también un factor a tener en cuenta, mas aun si tenemos en cuenta el pronóstico de aumento sostenido de las enfermedades crónicas ${ }^{(21) .}$

El estudio tuvo limitaciones, pues además de tratarse de un estudio descriptivo, la información fue obtenida del registro en las historias clínicas, con lo que pudiera haberse consignado información incompleta de algunos pacientes o registros clínicos con inconsistencias en la clasificación. Un punto importante por resaltar es el asociado a las múltiples enfermedades de los pacientes, lo que obligo a agrupar estas afecciones por trastornos clínicos y por sistemas, como se hizo en trabajos previos ${ }^{22,5,6,22)}$. Asimismo, se puede generar controversias asociadas a la aplicación por vía telefónica para EmR para la valoración funcional, sin embargo, se encontró que Bruno A. et al., determinaron la confiabilidad de la aplicación de la EmR en el seguimiento de pacientes con accidente cerebrovascular al alta, con adecuada fiabilidad en los resultados (17). Además, ha sido demostrada su utilidad como medición pronóstica en estudios multicéntricos ${ }^{(13,16,20)}$.

La aplicación de la ECG en pacientes sin traumatismo craneoencefálico pudo generar sesgos en la interpretación de datos, sin embargo, esta clasificación se ha aplicado a otros cuadros neurológicos como ictus en general, hemorragia intraparenquimal, hemorragia subaracnoidea y coma de etiología no traumática, pero, a pesar de sus limitaciones, se acepta que la escala de coma de Glasgow constituye un buen predictor de mortalidad intrahospitalaria y un instrumento útil para el triaje previo a la hospitalización ${ }^{(2,10,23)}$. Una limitación adicional, estuvo en la forma de calificación de la funcionalidad y valoración de la ECG, las que por la naturaleza del estudio, no pudieron ser realizadas por dos observadores y la evaluación consensuada o verificada el grado de concordancia. Otra situación que considerar es la realización del estudio en un solo hospital, podría generar un sesgo de selección y el tamaño muestral relativamente pequeño, pudo no ser suficiente para detectar causas raras o afinar la detección de mortalidad en pacientes jóvenes.

También es importante resaltar las fortalezas del estudio: la más importante lo constituye el seguimiento, intra y extrahospitalario. El tamaño muestral que fue calculado para evaluar mortalidad y asegurar la identificación de las enfermedades asociadas más frecuentes. Su realización basada en una ambiente hospitalario público, incluyó a todos los pacientes de un periodo determinado y permite mostrar lo que acontece en la realidad. Una bondad adicional lo constituye el ser el primer estudio de esta naturaleza en el Perú y en la región, lo que permitirá ser referencial y servir de base para la realización de estudios en otros centros hospitalarios, y así acumular una muestra mayor que promoverá el conocimiento real de la magnitud del problema, ello 'permitirá entender lo que representa como pronóstico así como identificar a la población de riesgo, de ese modo inspirar estudios sobre intervenciones que eviten muertes $\mathrm{y}$, por lo tanto, cambiar positivamente el ominoso pronóstico que tendrían actualmente las personas que acuden por alteración del estado mental a la emergencia.

Se concluye que la alteración del estado mental es una condición frecuente en la admisión por 
emergencia, que tiene una mortalidad que dobla la media hospitalaria, pues provoca la muerte en 1 de cada 4 personas afectadas a los tres meses, que se asocia predominantemente a situaciones prevenibles y reversibles como son las enfermedades infecciosas y en la que el compromiso severo de la funcionalidad al ingreso y alta hospitalaria, constituyen los más importantes predictores independientes de mortalidad.
Contribuciones de autoría: CMAAD y GMR, participaron en la concepción y diseño del estudio, CMAAD, MLP, JLH participaron de la recolección, análisis e interpretación de resultados y redacción del artículo. Todos los autores aprobaron la versión final

Conflictos de interés: los autores declaran no tener conflictos de interés.

Fuentes de financiamiento: autofinanciado

\section{REFERENCIAS BIBLIOGRÁFICAS}

1. American College of Emergency Physicians. Clinical policy for the initial approach to patients presenting with altered mental status. Ann Emerg Med. 1999 Feb;33(2):251-81.

2. Kanich W, Brady WJ, Huff JS, Perron $\mathrm{AD}$, Holstege C, LindbeckG, et al. Altered mental status: evaluation and etiology in the ED. Am J Emerg Med. 2002 Nov;20(7):613-7.

3. Ropper A. Estados confusionales agudos y coma. Cap. 257. En: Longo DL, Kasper DL, Jameson JL, Fauci AS, Hauser SL, Loscalzo J, Eds. Harrison Principios de Medicina Interna. 18a ed. Madrid: McGrawHill; 2006.

4. Martínez Díaz MV, Sáez Méndez L. Alteraciones del nivel de conciencia. Medicine. 2007;9(87):5585-91.

5. Pei-Fang L, Giou-Teng Y, MingJen T, Sheng-Chuan H. Analysis of patients with altered mental status in an emergency department of Eastern Taiwan. Tzu Chi Med J. 2009;21(2):151-5.

6. Lim Beng Leong, Kenneth Heng Wei Jian, Alicia Vasu, EillyneSeow. Prospective study of patients with altered mental status: clinical features and outcome. Int J Emerg Med. 2008;1(3):179-82. doi: 10.1007/ s12245-008-0049-8.

7. Slattery DE, Pollack CV Jr. Seizures as a cause of altered mental status. Emerg Med Clin North Am. 2010 Aug;28(3):517-34. doi: 10.1016/j. emc.2010.03.011.

8. Angel MJ, Young GB. Metabolic encephalopathies. Neurol Clin. 2011 Nov;29(4):837-82. doi: 10.1016/j. ncl.2011.08.002..

9. O’Donnell MJ, Xavier D, Liu L, Zhang H, Chin SL, Rao-Melacini P, et al. Risk factors for ischemic and intracerebral haemorrhagic stroke in
22 countries (the INTERSTROKE study): a case-control study. Lancet. 2010 Jul 10;376(9735):112-23. doi: 10.1016/S0140-6736(10)60834-3.

10. de Castro P. Paciente con alteración de conciencia en urgencias. An Sist Sanit Navar. 2008;31 Suppl 1:87-97.

11. Gabbe BJ, Cameron PA, Finch CF. The status of the Glasgow Coma Scale. Emerg Med (Fremantle). 2003 Aug;15(4):353-60.

12. Laureys S, Owen AM, Schiff ND. Brain function in coma, vegetative state, and related disorders. Lancet Neurol. 2004 Sep;3(9):537-46.

13. van Swieten JC, Koudstaal PJ, Visser MC, Schouten HJ, van Gijn J. Interobserver agreement for the assessment of handicap in stroke patients. Stroke. 1988 May;19(5):604-7.

14. Koita J, Riggio S, Jagoda A. The mental status examination in emergency practice. Emerg Med Clin North Am. 2010 Aug;28(3):439-51. doi: 10.1016/j.emc.2010.03.008.

15. Rankin J. Cerebral vascular accidents in patients over the age of 60 . II: Prognosis. Scott Med J. 1957 May;2(5):200-15.

16. Banks JL, Marotta CA. Outcomes validity and reliability of the modified rankin scale: implications for stroke clinical trialsa literature review and synthesis. Stroke. 2007 Mar;38(3):1091-6

17. Bruno A, Akinwuntan AE, Lin C, Close B, Davis K, Baute V, et al. Simplified modified rankin scale questionnaire: reproducibility over the telephone and validation with quality of life. Stroke. 2011 Aug;42(8):2276-9. doi: 10.1161/ STROKEAHA.111.613273.

18. Tello-Rodríguez T; Varela-Pinedo L, Ortiz-Saavedra J, Chávez-Jimeno $\mathrm{H}$.
Estancia hospitalaria y mortalidad en adultos mayores hospitalizados en un hospital general de Lima Metropolitana, 1997-2008. Rev Med Hered. 2011;22(1):23-8.

19. Rabinstein AA, McClelland RL, Wijdicks EF, Manno EM, Atkinson JL. Cardiopulmonary resucitation in critically ill neurologic neurosurgical patients. Mayo Clin Proc. 2004 Nov;79(11):1391-5.

20. Wolfe CD, Taub NA, Woodrow J, Richardson E, Warburton FG, Burney PG. Patterns of acute stroke care in three districts of southern England. J Epidemiol Community Health. 1993 Apr;47(2):144-8.

21. Fisher-Hoch SP, Mathews CE, McCormick JB. Obesity, diabetes and pneumonia: the menacing interface of non-communicable and infectious diseases. Trop Med Int Health. 2013 Dec;18(12):1510-9. doi: 10.1111/ tmi.12206.

22. Zonta MB, Almeida SM, Carvalho MT, Werneck LC. Evaluation of AIDS-Related Disability in a General Hospital in Southern, Brazil. Braz J Infect Dis. 2005 Dec;9(6):479-88. Epub 2006 Jan 9.

23. Hai-yu X, Yu-xuan W, Teng-da X, Hua-dong Z, Shu-bin G, Zhong $\mathrm{W}$, et al. Evaluation and treatment of altered mental status patients in the emergency department: Life in the fast lane. World J Emerg Med. 2012;3(4):270-7.

Correspondencia: Germán Málaga

Dirección: Calle Chavin 159. CC Monterrico. Santiago de Surco. Lima 33. Perú

Teléfono: +51992768300

Correo electrónico:german.malaga@upch.pe 